\title{
Robot-Assisted Thoracic Surgery in Non-small Cell Lung Cancer
}

\author{
Jun Hee Lee, M.D., Jeong In Hong, M.D., Hyun Koo Kim, M.D., Ph.D. \\ Department of Thoracic and Cardiovascular Surgery, Korea University Guro Hospital, Korea University College of Medicine, Seoul, Korea
}

\author{
ARTICLE INFO \\ Received June 22, 2021 \\ Accepted July 16, 2021 \\ Corresponding author \\ Hyun Koo Kim \\ Tel 82-2-2626-3106 \\ Fax 82-2-866-6377 \\ E-mail kimhyunkoo@korea.ac.kr \\ ORCID \\ https://orcid.org/0000-0001-7604-4729
}

\begin{abstract}
Lobectomy is the standard treatment for early non-small cell lung cancer. Various surgical techniques for lobectomy have been developed, and minimally invasive thoracic surgery, such as video-assisted thoracic surgery or robot-assisted thoracic surgery, has been considered as an alternative to conventional open thoracotomy. The recently robotic lobectomy technique has developed since the first case series was published in 2002. Several studies have reported that robotic lobectomy has comparable oncologic and perioperative outcomes to those of video-assisted thoracic surgery lobectomy and open lobectomy. However, robotic lobectomy remains a challenge for surgeons because of the steep learning curve, reduced tactile sensation, difficulty in port placement, and challenges in cooperation between the surgeon and assistant. Many studies have reported on robotic lobectomy, but few have presented surgical techniques for robotic lobectomy. In this article, the surgical techniques and optimal performance of robotic lobectomy are described in detail for all 5 types of lobectomy for surgeons beginning with robotic lobectomy.
\end{abstract}

Keywords: Robot assisted thoracic surgery, Robotic lobectomy, Lobectomy, Non-small cell lung cancer

\section{Introduction}

Lobectomy, which involves the anatomical resection of an entire lobe of the lung, is the standard treatment for early non-small cell lung cancer (NSCLC). Traditionally, it was performed via an open thoracotomy approach, which is associated with severe postoperative pain and a large wound. Minimally invasive thoracic surgery lobectomy, including video-assisted thoracic surgery (VATS) and robotic lobectomy, has been considered an appropriate approach and is associated with low perioperative morbidity, less pain, shorter hospitalization, and equivalent oncologic outcomes compared with open thoracotomy [1-5].

Since the first report of VATS lobectomy in the early 1990s [6], it has become a popular surgical approach [7-9]. However, several disadvantages of VATS lobectomy, such as the lack of flexibility, difficulty of hand-eye coordination, and limitations of 2-dimensional imaging, led to the development of robotic lobectomy as a novel technique [10]. Robotic lobectomy, which is performed with a robotic surgical system, offers improved 3-dimensional imaging, hand-eye coordination, freedom of movement, and tremor filtration, allowing safe surgical procedures [11,12].

Since the first case series on robotic lobectomy, reported in 2002 [13], it has gradually developed with technological innovations of robotic systems. The introduction of a robotic stapler (EndoWrist Stapler; Intuitive Surgical Inc., Sunnyvale, CA, USA) in the da Vinci Xi has made it possible for total robotic lobectomy to be directly controlled by surgeons, and may reduce the operative time $[12,14,15]$.

Several studies have reported the advantages of robotic lobectomy, which is associated with a significant reduction in the length of hospitalization, chest tube drainage duration, postoperative complications, and mortality when compared with the open thoracotomy approach [2,16-19]. Two studies using propensity score matching for the outcomes of open, VATS, and robotic lobectomy demonstrated no significant differences in morbidity and oncologic outcomes [20,21].

In recent years, robotic lobectomy has been indicated for more challenging cases owing to its superior maneuverability. Qiu et al. [22] reported a successful case of robotic double-sleeve lobectomy and demonstrated its ability to be used for bronchoplasty and angioplasty. Wilson et al. [23] 
reported the prevalence of nodal upstaging during robotic lobectomy in early NSCLC; therefore, robotic mediastinal lymph node dissection (MLND) may be useful in patients with locally advanced N2 NSCLC due to improved dexterity and visualization.

However, robotic lobectomy remains a challenge because of its steep learning curve, reduced tactile sensation, difficulty in port placement, and challenges in cooperation between the surgeon and assistant. Robotic lobectomy techniques have many variations in terms of the number of incisions, number of robotic arms used, use of a utility incision, site location for port placement, and use of $\mathrm{CO}_{2}$ insufflation. These variations add to the difficulty of the procedure.

In this article, the surgical techniques and optimal performance of robot lobectomy are described in detail for all 5 types of lobectomy for surgeons beginning to perform robotic lobectomy, along with useful tips on how to overcome procedure-related problems.

\section{Indications and contraindications of robotic surgery}

The indications for robotic lobectomy are similar to those for conventional lobectomy, although they may be more extensive than those of VATS and open lobectomy. Robotic lobectomy may be indicated in patients with marginal pulmonary function and locally advanced cancer $[24,25]$. Generally, some conditions such as dense adhesions, bronchial invasion, chest wall invasion, hilar nodal invasion, preoperative radiotherapy, prior thoracic surgery, and hilar nodal invasion are no longer regarded as absolute contraindications; instead, they are considered relative contraindication $[4,26]$. In our opinion, robotic lobectomy by an experienced surgeon is feasible in these patients.

\section{Learning curve of robotic surgery}

Although defining the learning curve is difficult, several studies have estimated the learning curve for a robotic lobectomy to be 18-32 cases (Table 1). According to some studies, at least 20 cases are required for surgical competence $[18,27,28]$. Arnold et al. [29] reported a learning curve of 22 cases, while Song et al. [30] reported it as 32 cases for the surgeon and 20 cases for the bedside assistant.

Few studies have compared the learning curves of robotic lobectomy and VATS lobectomy. Jang et al. [31] reported the outcomes of their experiences of performing robotic lobectomy with VATS lobectomy. They compared the outcomes of their early experience of robotic lobectomy $(n=40)$ with the outcomes of initial $(n=40)$ and recent VATS lobectomy $(\mathrm{n}=40)$. The complication rates $(10 \%$ versus $32.5 \%$, $\mathrm{p}=0.027)$, intraoperative bleeding ( $219 \mathrm{~mL}$ versus $374 \mathrm{~mL}$, $\mathrm{p}=0.017)$, and the median length of postoperative stay (6 days versus 9 days, $\mathrm{p}<0.001$ ) was significantly lower in the robotic lobectomy group than in the initial VATS group.

In the future, robotic surgery training programs consisting of simulated skills training, dry lab-based learning, and video articles can help shorten this learning curve.

\section{Operative technique}

\section{General considerations}

A double-lumen tube is placed for one-lung ventilation, and the patient is positioned in the lateral decubitus position. The operating table is flexed to widen the intercostal space (ICS) and to move the hip down. If possible, the patients are placed in the reverse Trendelenburg position. Port mapping is performed according to the use of the utility incision, the location of the lobe to be resected, and the number of ports required. After an appropriate incision, the robotic trocar and working port are placed. In patients with a narrow thoracic cavity or short stature, a single port with multi-instrument access (Lapsingle; Sejong Medical, Paju, Korea) is used at our center with $\mathrm{CO}_{2}$ insufflation (maximum 6-10 $\mathrm{mm} \mathrm{Hg}$ ), which facilitates lung collapse and caudal shift of the diaphragm.

Table 1. The learning curve in robotic lobectomy

\begin{tabular}{|c|c|c|c|c|}
\hline Study & Year & Surgical technique & $\begin{array}{l}\text { Total no. of patients who } \\
\text { underwent robotic lobectomy }\end{array}$ & $\begin{array}{l}\text { No. of operations required to } \\
\text { achieve surgical competence }\end{array}$ \\
\hline Melfi et al. [27] & 2008 & 4 ports, 4 arms & 107 & 20 \\
\hline Gharagozloo et al. [28] & 2009 & 3 ports, 3 arms & 100 & 20 \\
\hline Veronesi et al. [18] & 2010 & 4 ports, 4 arms & 54 & 20 \\
\hline Arnold et al. [29] & 2019 & 5 ports, 4 arms & 101 & 22 \\
\hline Song et al. [30] & 2019 & 4 ports, 3 arms & 208 & $\begin{array}{l}32 \text { (for surgeon) } \\
20 \text { (for assistant) }\end{array}$ \\
\hline
\end{tabular}


Before robot docking, a careful thoracoscopic examination is required to identify the pleural adhesion, pleural effusions, and other metastases. We routinely place the vessel loop and rolled-up gauze in the fissure before the introduction of instrument arms to save time (Fig. 1A). The use of a vessel loop for retraction of the vessel branches and bronchus facilitates stapling and prevents vascular injury (Fig. 1B). The placement of rolled-up gauze between the robot wrist and the structure that needs to be retracted facilitates dissection without the fourth robotic arm or the assistant at the bedside, especially for the hilar and nodal tissues (Fig. 1C).

The robotic endoscope is always installed centrally, and other devices are installed depending on the surgeon's preference. A Cadiere grasper is installed on the left, and a Maryland bipolar forceps is installed on the right. Most vessels, as well as the bronchi, are divided with the robotic stapler (EndoWrist Stapler; Intuitive Surgical Inc., Sunnyvale, CA, USA) in the da Vinci Xi surgical system, and small vessel branches can be ligated with robotic Hem-oLok clips.

Robotic MLND is routinely performed in all patients. Complete dissection of the hilar lymph nodes is a key point in robotic lobectomy. It is always optimal to remove all nodal tissues around and among hilar structures, because doing so helps to identify the anatomy and removing these nodal tissues makes it easy to pass through the stapler. The cut end of the surgical glove is used as a retrieval bag, and the resected lymph nodes are retrieved at the cut end of the surgical glove to avoid contamination of the wound and thoracic cavity with cancer cells (Fig. 1D).

At the end of the procedure, the lung specimens are removed using a specimen bag through the utility incision. We routinely perform a paravertebral intercostal nerve block, which is an intrapleural administration of bupiva- caine via the intrathoracic approach under endoscopic visualization. Before closing the wound incision, a $24 \mathrm{~F}$ or $20 \mathrm{~F}$ chest tube is inserted into the incision.

\section{Port mapping}

Optimal port placement is a vitally important component of the success of robotic lobectomy, as it avoids compression injuries, allows free mobility of the robotic instruments, and facilitates safe surgery. Currently, robotic lobectomy is performed using 3-4 arms with 2-4 ports, with the exception that a few surgeons use the 5-port approach. When using a robotic stapler, creating a $12-\mathrm{mm}$ port for the stapler as low as possible can facilitate robotic arm maneuverability, because the end effector of the robotic stapler is longer than the other robotic instruments [32].

\section{The 4-port approach}

Veronesi et al. [18] and Pardolesi et al. [33] introduced 4-port robotic lobectomy, consisting of 3 trocar ports and 1 utility port. A 3- to $4-\mathrm{cm}$ utility incision is made at the fifth ICS anterior to the latissimus dorsi. A $12-\mathrm{mm}$ port incision (camera port) is made in the seventh ICS at the mid-axillary line for right-side lobectomy, while the incision site is $2 \mathrm{~cm}$ posterior for left-side lobectomy. An 8-mm port incision is made at the eighth ICS $8 \mathrm{~cm}$ posteriorly from the camera port, and the other $8-\mathrm{mm}$ port incision is made in the auscultatory triangle $[18,33]$.

Cerfolio et al. [34] introduced 4-port robotic lobectomy consisting of 3 ports and 1 assistant port. One $5-\mathrm{mm}$ port incision (videoscope) is made at the sixth or seventh ICS along the midaxillary line, and another $5-\mathrm{mm}$ port incision (robotic arm 3) is made at the seventh to eighth ICS, just anterior to the spinous process of the vertebral body. An $8-\mathrm{mm}$ port (robotic arm 2) is made at the seventh to
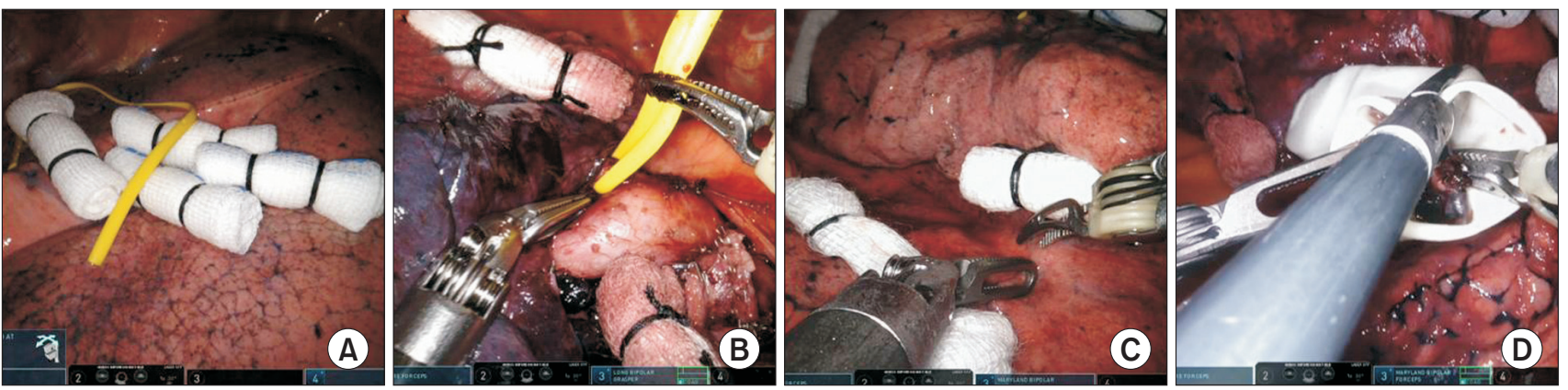

Fig. 1. Tips for robotic lobectomy. (A) Placement of rolled-up gauze and a vessel loop on the fissure. (B) Pulmonary artery isolated with a vessel loop. (C) Surgical field with traction using rolled gauze. (D) The cut end of the surgical glove as a retrieval bag for the resected lymph nodes. 
eighth ICS, $10 \mathrm{~cm}$ anterior to robotic arm 3. The camera port is made at the seventh to eighth ICS, $9 \mathrm{~cm}$ anterior to robotic arm 2. A $15-\mathrm{mm}$ assistant port is made at the 2 or 3 lower ribs [34].

\section{The 3-port approach}

A 3- to $4-\mathrm{cm}$ utility incision is made at the seventh or eighth ICS along the mid-axillary line, a $12-\mathrm{mm}$ port incision is made at the sixth or seventh ICS along the anterior axillary line, and the other $12-\mathrm{mm}$ port incision is made at the eighth or ninth ICS along the scapular line, $8 \mathrm{~cm}$ apart from each other. A single port with multi-instrument access (Lapsingle; Sejong Medical) is installed through the utility incision. An 8-mm trocar for the robotic camera is inserted through the working port, and the assistant inserts his or her instruments through the other side of the working port (Fig. 2).

\section{The 2-port approach}

A 3- to 4 -cm utility incision (camera and arm port 2 ) is made at the seventh or eighth ICS, along the posterior axillary line, and a 12 -mm port incision (arm port 1 ) is made at the sixth or seventh ICS along the anterior axillary line without an assistant port. A single port with multi-instrument access (Lapsingle; Sejong Medical) is installed through the utility incision. Two 8 - $\mathrm{mm}$ trocars for the robotic camera and robotic arm 1 (for a Cadiere grasper or Maryland bipolar forceps) are inserted through the work-

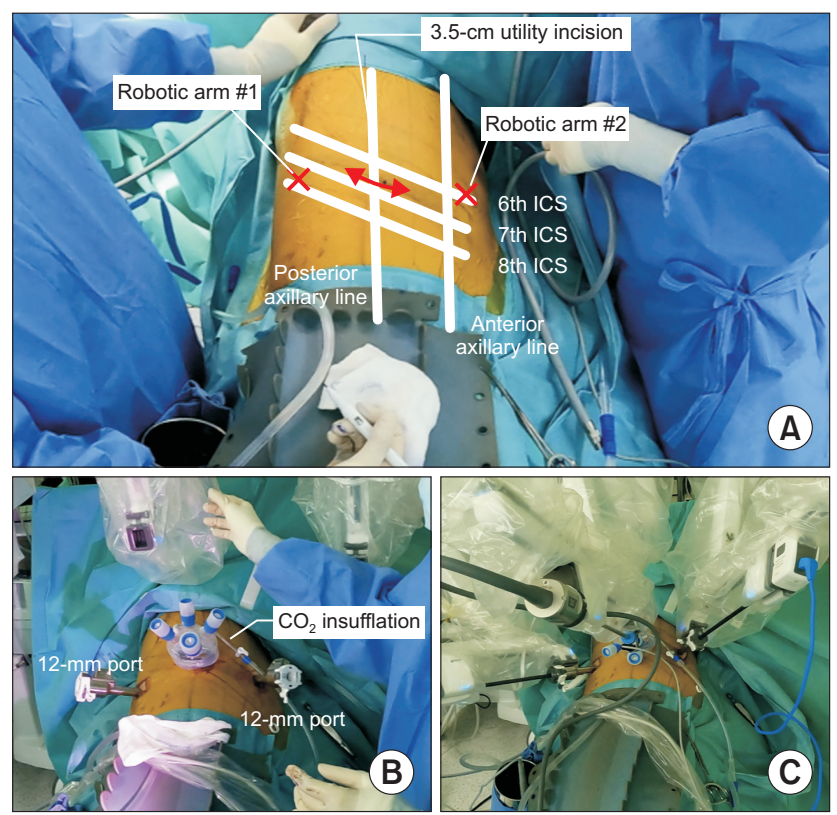

Fig. 2. (A-C) Photographs of port placement in the 3-port approach for robotic lobectomy. ICS, intercostal space. ing port. The $12-\mathrm{mm}$ trocars are inserted through another 12-mm port for robotic arm 2 (Cadiere grasper, Maryland bipolar forceps, or robotic EndoWrist staplers). The assistant inserts his or her instruments through the space between 2 trocars in the working port (Fig. 3)

\section{The 5 types of lobectomy}

Below are descriptions of how to perform each type of lobectomy.

\section{Right upper lobectomy}

The dissection of the hilar structures should be performed individually, and lymphadenectomy should also be performed. The anatomical structures are carefully identified (especially in the posterior ascending artery). The surgical approach differs depending on the completeness of the pulmonary fissure.

When the fissure is fused and not well developed, the hilar approach is preferred to dissect the mediastinal pleura over the anterior hilum. The sequence of dissection is anterior to posterior in a single direction, as shown in Fig. 4: (1) isolation and division of the right upper lobe (RUL) vein, (2) isolation and division of the anterior trunk of the pulmonary artery, (3) isolation and division of posterior ascending artery, (4) isolation and division of the RUL bron-
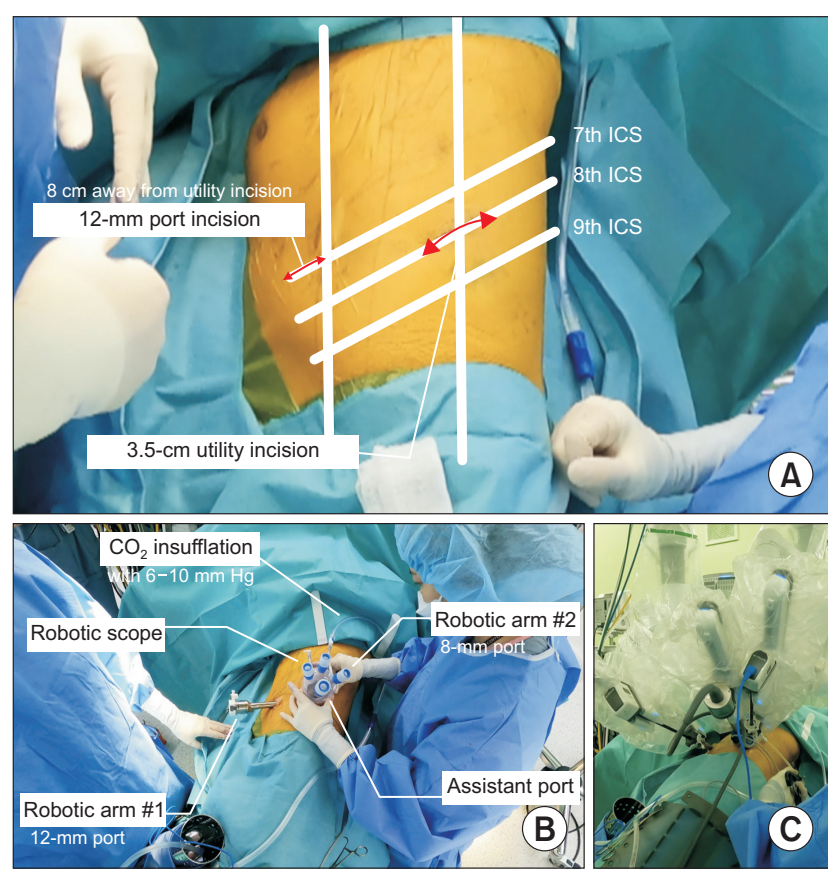

Fig. 3. (A-C) Photographs of port placement in the 2-port approach for robotic lobectomy. ICS, intercostal space. 

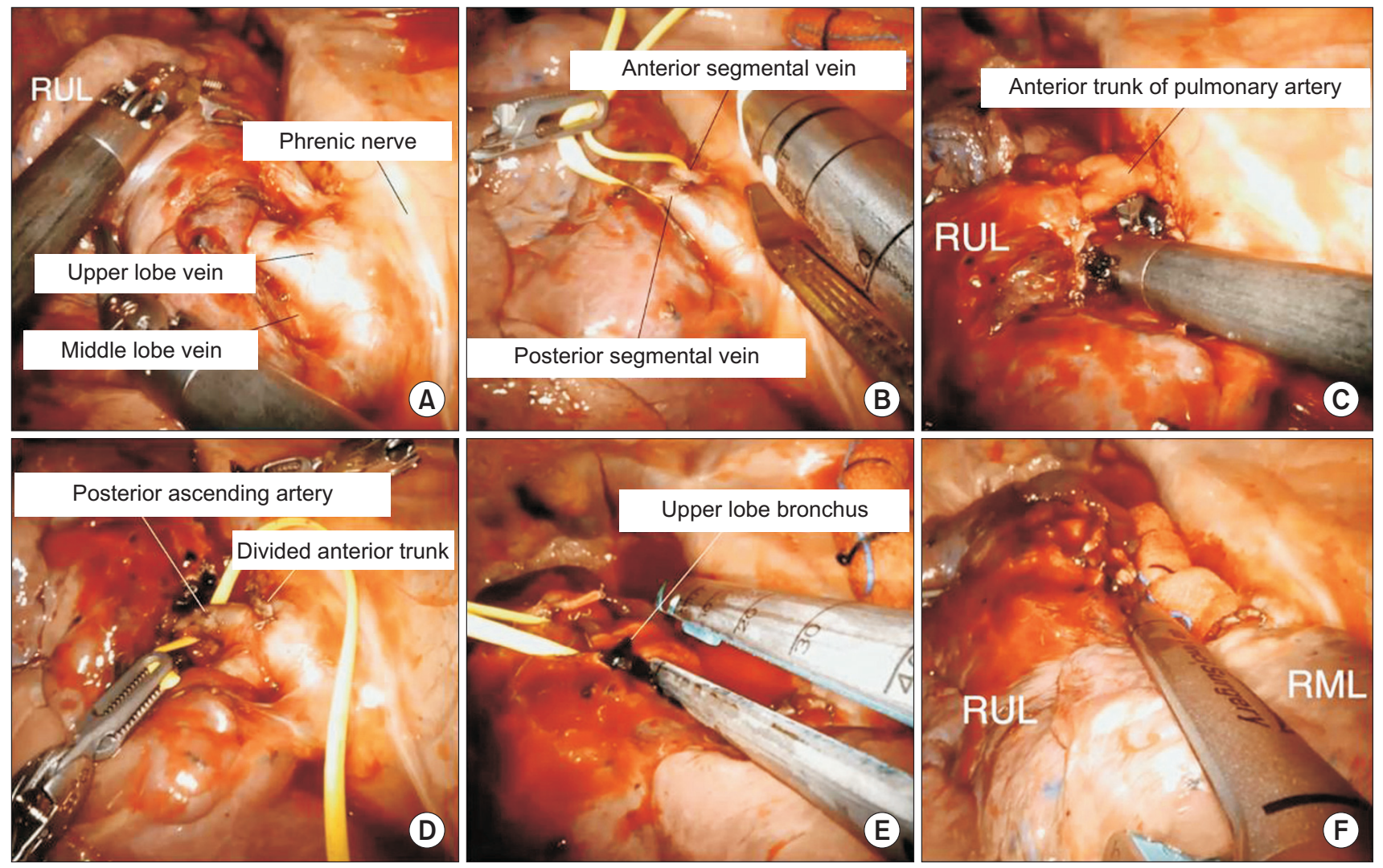

Fig. 4. Robotic right upper lobectomy for patients with a fused fissure. (A) Identification of the bifurcation between the middle lobe vein and upper lobe vein. (B) Division of the upper lobe vein. (C) Isolation of the anterior trunk of the pulmonary artery. (D) Isolation of the posterior ascending artery. (E) Division of the upper lobe bronchus. (F) Completion of the fissure. RUL, right upper lobe; RML, right middle lobe.

chus, and (5) completion of the fissure. The sequence of steps 1 and 2 can be changed.

Step 1: Isolation and division of the RUL vein

The RUL is retracted posteriorly to expose the upper hilar region and the anterior mediastinum, and the anterior mediastinum and anterior mediastinal pleura are opened to expose the upper lobe vein. Once the upper lobe vein is identified, dissection is continued until the bifurcation between the middle lobe vein and upper lobe vein is identified. The anterior segmental vein and posterior segmental vein are encircled with a vessel loop and divided with a robotic stapler.

Step 2: Isolation and division of the anterior trunk of the pulmonary artery

After division of the upper lobe vein, the hilum is carefully dissected to expose the pulmonary artery. The connective tissue and nodal tissue around the pulmonary artery are removed to expose the anterior trunk of the pulmonary artery. The anterior trunk is isolated from the main pulmonary artery, and the grasper is passed behind the anterior trunk to ensure sufficient space for the stapler's passage. The anterior trunk of the pulmonary artery is encircled with a vessel loop and divided using a robotic stapler.

Step 3: Isolation and division of the posterior ascending artery

Division of the posterior ascending artery can be performed before or after division of the RUL bronchus. The posterior ascending artery is encircled with a vessel loop and divided with robotic stapler. When the posterior ascending artery is too small, robotic Hem-o-Lok clips can be used to ligate it.

Step 4: Isolation and division of the RUL bronchus

Complete dissection of the lymph nodes should be performed, which facilitates the passage of the stapler. The upper lobe is retracted anteriorly, and the posterior aspect 
of the hilum is dissected. The dissection is continued to expose the RUL bronchus and bronchus intermedius. The upper lobe bronchus is encircled with a vessel loop and divided with a robotic stapler.

\section{Step 5: Completion of the fissure}

To complete the fissure, it is usually divided with a robotic stapler. Care is taken to identify the main pulmonary artery and right middle lobe (RML) artery. Next, the speci- men is removed using a specimen bag through the utility incision.

However, when the fissure is complete and well developed, we prefer to perform the steps in the following order (Fig. 5): (1) isolation and division of the posterior ascending artery, (2) dissection of the pleura at the RUL bronchus and bronchus intermedius bifurcation, (3) isolation and division of the anterior trunk of the pulmonary artery, (4) isolation and division of the RUL bronchus, and (5) isola-
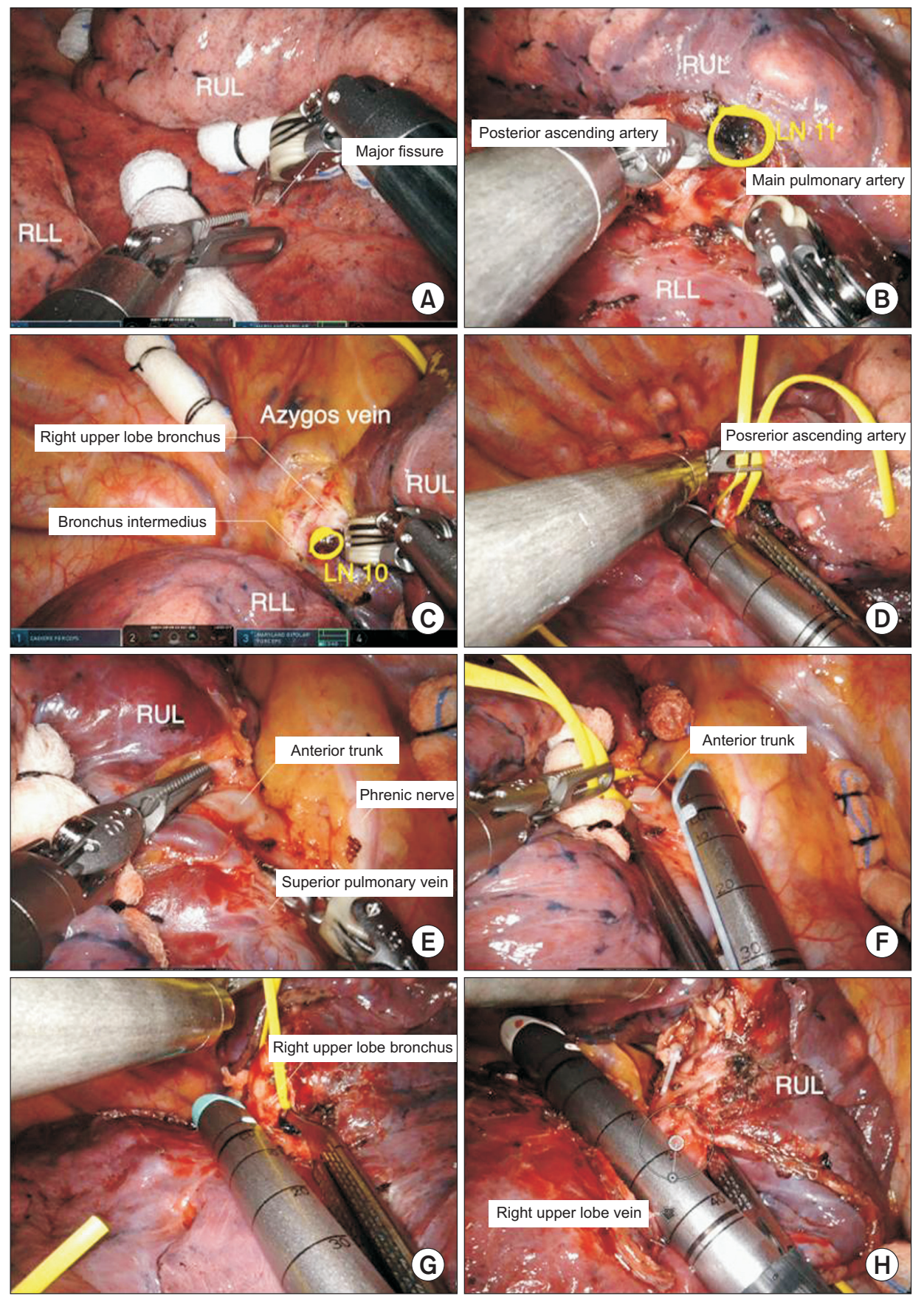

Fig. 5. Robotic right upper lobectomy for patients with a complete fissure. (A) Dissection of the major fissure. (B) Identification of the posterior ascending artery. (C) Dissection of the pleura at the right upper lobe (RUL) bronchus and bronchus intermedius bifurcation. (D) Division of the posterior ascending artery. (E) Identification of the anterior trunk of pulmonary artery and superior pulmonary vein. (F) Division of the anterior trunk of pulmonary artery. (G) Division of the upper lobe bronchus. $(\mathrm{H})$ Division of the upper lobe vein. LN, lymph node. 
tion and division of the RUL vein.

Step 1: Isolation and division of the posterior ascending artery

The RUL is retracted superiorly, and the right lower lobe (RLL) is retracted inferiorly to expose the pulmonary artery. The major fissure between the RUL and RLL is dissected using long bipolar forceps, and the nodal tissues are removed to identify the anatomy of the pulmonary artery. Once the posterior ascending artery is exposed, the dissection continues until the main pulmonary artery is identified. The incomplete major fissure between the RUL and RLL can be divided with a robotic stapler to expose the pulmonary artery. Sometimes it is better to divide the major fissure last to reduce air leakage. The posterior ascending artery is encircled with a vessel loop and divided using a robotic stapler. If the posterior ascending artery is too small, robotic Hem-o-Lok clips can be used to ligate it.

Step 2: Dissection of the pleura at the RUL bronchus and bronchus intermedius bifurcation

The lung is retracted inferiorly to expose the superior surface of the hilum, and the upper hilar region is dissected. This prepares the field for the isolation of the RUL bronchus. Once the connective and nodal tissues are removed, the right main bronchus and the bronchus intermedius are clearly exposed.

Step 3: Isolation and division of the anterior trunk of the pulmonary artery

The lung is retracted posteriorly, and the upper hilar region is dissected anteriorly to posteriorly. Once the anterior trunk is exposed, it should be separated from the superior pulmonary vein. The anterior trunk is encircled with a vessel loop and divided using a robotic stapler.

Step 4: Isolation and division of the RUL bronchus

After division of the anterior trunk, the RUL bronchus is exposed, and nodal tissues around the bronchus should be removed. The RUL bronchus is encircled with a vessel loop and divided using a robotic stapler.

Step 5: Isolation and division of the RUL vein

The bifurcation between the RML vein and upper lobe vein should be dissected to identify anomalies of the venous anatomy. Before division of the RUL vein, the incomplete minor fissure between the RUL and RML is usually divided using a robotic stapler. The RUL is retracted superiorly, and the RUL vein is divided using a robotic stapler.
Next, the specimen is removed using a specimen bag via the utility incision.

\section{Right middle lobectomy}

Although there are many variations in the techniques of right middle robotic lobectomy, we prefer to perform the procedure in the following order (Fig. 6): (1) division of the major fissure, (2) division of the middle lobe vein, (3) division of the middle lobe bronchus, (4) division of the middle lobe artery, and (5) division of the minor fissure.

Complete dissection of the hilar lymph nodes is the key element of robotic right middle lobectomy because of the narrow space between structures, and it facilitates the identification of the middle lobe bronchus and middle lobe artery. The phrenic nerve is carefully identified.

Step 1: Dissection and division of the major fissure

We usually prefer to dissect the anterior portion of the major fissure first with long bipolar forceps. The interlobar lymph nodes are carefully removed to facilitate the identification of the pulmonary artery, bronchus, and vein. Once the artery to the RLL is identified, a tunnel for the division of the major fissure can be created. Part of the incomplete major fissure can be divided using a robotic stapler.

Step 2: Isolation and division of the RML vein

Once the major fissure is divided, the pulmonary vein is well exposed. After retraction of the lung posteriorly, the anterior aspect of the hilum is dissected. Although the middle lobe vein most often joins the superior pulmonary vein, it may join the right inferior pulmonary vein and directly drain into the atrium. After identification of the RUL vein and RML vein, the RML vein is encircled with a vessel loop and divided with a robotic stapler.

Step 3: Isolation and division of the middle lobar bronchus

The middle lobe bronchus is always located below the middle lobe artery. The middle lobe bronchus is dissected and complete dissection of the lymph node should be performed before closing and dividing by the stapler. The middle lobar bronchus is encircled with a vessel loop and divided using a robotic stapler. After clamping the bronchus and before firing the stapler, ventilation of the right lung can confirm the exact position of the stapler. The minor fissure can be divided using a robotic stapler before or after division of the middle lobe artery.

Step 4: Isolation and division of the middle lobe artery

The nodal tissues should be dissected completely to iden- 

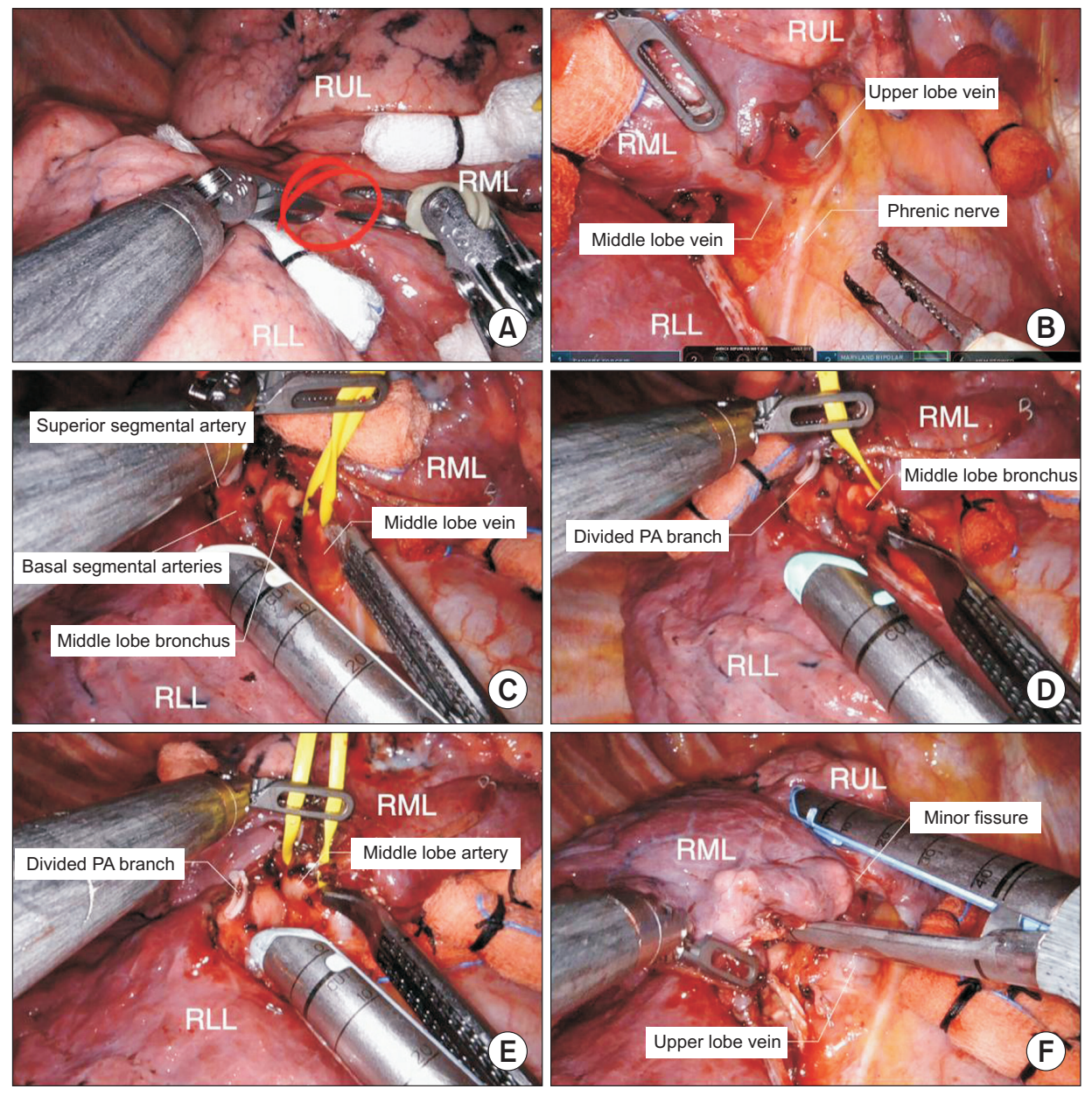

Fig. 6. Robotic right middle lobectomy. (A) Dissection of the major fissure. (B) Identification of the bifurcation between the middle lobe vein and upper lobe vein. (C) Division of the middle lobe vein. (D) Division of the middle lobe bronchus. (E) Division of the middle lobe artery. (F) Completion of the minor fissure. RUL, right upper lobe; $R M L$, right middle lobe; RLL, right lower lobe; PA, pulmonary artery.

tify the anatomy of the pulmonary artery. A4 and A5 can arise as separate branches (In 50\% of cases) or a common trunk (In the other $50 \%$ of cases) from the interlobar artery. The middle lobe artery is encircled with a vessel loop and divided using a robotic stapler. Once all the hilar structures are divided, the minor fissure between the RUL and RML is usually divided using a robotic stapler. Next, the specimen is removed using a specimen bag via the utility incision.

\section{Right lower lobectomy}

The inferior pulmonary ligament is usually dissected first to approach the inferior pulmonary vein. Complete dissection of the lymph nodes can open the mediastinal space and enable safer dissection of the artery, vein, and bronchi.

The surgical approach differs depending on the completeness of the pulmonary fissure. When the fissure is fused and not well developed, we prefer to perform the steps in the following order (Fig. 7): (1) division of the infe- rior pulmonary ligament, (2) isolation and division of the inferior pulmonary vein, (3) isolation and division of the lower lobe artery, and (4) isolation and division of the lower lobe bronchus.

If the fissure is well developed, we prefer a caudal approach, which is described in the section dealing with left lower lobectomy.

Step 1: Division of the inferior pulmonary ligament

The lung is retracted superiorly using Cadiere forceps, and the surgeon begins to divide the inferior edge of the inferior pulmonary ligament using long bipolar forceps. An assistant can help retract the lung superiorly using a long sponge stick. The inferior pulmonary ligament is divided with all surrounding tissues, and lymph node stations 7,8 , and 9 can be removed.

Step 2: Isolation and division of the inferior pulmonary vein

Next, the lung is retracted anteriorly to expose the subcarinal area using the rolled-up gauze to avoid direct 

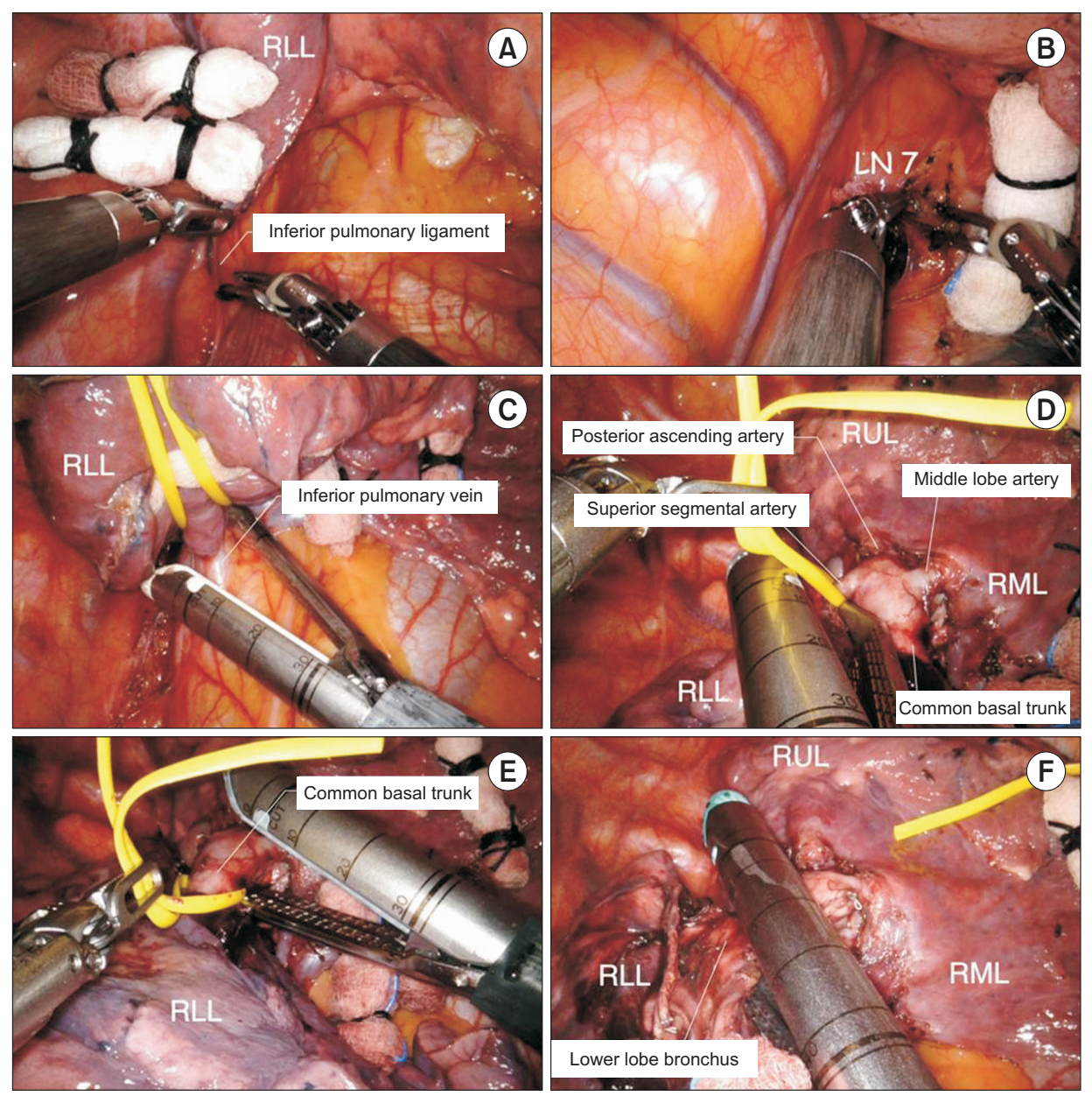

Fig. 7. Robotic right lower lobe (RLL) lobectomy. (A) Division of the inferior pulmonary ligament. (B) Dissection of lymph node (LN) station 7. (C) Division of the inferior pulmonary vein. (D) Division of the superior segmental artery. (E) Division of the common basal trunk. (F) Division of the lower lobe bronchus. RUL, right upper lobe; RML, right middle lobe. grasping. After dissection of the subcarinal lymph node, the RLL is retracted posteriorly, and the inferior pulmonary vein is carefully dissected. The mediastinal pleura should be completely dissected to identify the middle lobe vein and the right inferior pulmonary vein. The inferior pulmonary vein is encircled with a vessel loop and then divided using a robotic stapler.

Step 3: Isolation and division of the lower lobe artery

The anterior part of the major fissure between the RLL and the RML is dissected using long bipolar forceps, and the hilar lymph nodes are removed. We prefer to divide the fused major fissure between the RLL and the RML using a robotic stapler to achieve better exposure of the lower lobe artery in the interlobar space. Complete dissection should be continued until the superior segmental and ascending posterior arteries are identified.

Once the superior segmental artery and basal trunk are exposed, they can be divided in 1 step or 2 steps. We prefer a 2-step division, in which the superior segmental artery is divided first with a robotic stapler, and then the basal trunk is divided with a robotic stapler.

Step 4: Isolation and division of the lower lobe bronchus

After division of the pulmonary artery, all hilar lymph nodes should be removed. The lower lobe bronchus is divided using a robotic stapler. Compromising the middle lobe bronchus should be carefully avoided. Next, the specimen is removed using a specimen bag via the utility incision.

\section{Left upper lobectomy}

The left upper lobe (LUL) pulmonary branches may arise in a fairly random order, and they frequently form small trunks of 2 or more branches. Some branches are too small and short to place the stapler. Therefore, careful dissection of the vessel and placement of the stapler must be performed to avoid vessel laceration. Some branches of the 
pulmonary artery are too small and short, and they can be ligated with robotic Hem-o-Lok clips.

Although the sequence of steps in dissection may vary to some degree due to variations in anatomy, we prefer to perform the steps in the following order (Fig. 8): (1) isolation and division of the superior pulmonary vein, (2) division of the lingular segmental arteries and posterior pulmonary artery branches, (3) isolation and division of the LUL bronchus, and (4) isolation and division of the apicoposterior segmental artery

Step 1: Isolation and division of the superior pulmonary vein

The lung is retraced posteriorly to expose the hilum, and the anterior mediastinal pleura is opened. Dissection should be continued until the bifurcation between the superior pulmonary vein and the inferior pulmonary vein is identified. The division of the left superior pulmonary vein is easier than that of the RUL vein due to the absence of the RML vein. The superior pulmonary vein is encircled with a vessel loop, and then divided using a robotic stapler.
Step 2: Division of the lingular segmental arteries and posterior pulmonary artery branches

Next, we dissect the fissure using long bipolar forceps. Retraction of the lung by the robotic wrist with rolled-up gauze facilitates dissection of the fissure. After the fissure dissection, the anatomy of the pulmonary artery is identified. Once the superior segmental artery is identified, a tunnel for fissure division can be created. The fused anterior part of the major fissure can be divided using a robotic stapler through the tunnel. The nodal tissues are dissected to facilitate the subsequent passage of the robotic stapler. Once the entire interlobar artery is clearly exposed, the posterior pulmonary artery branches and lingular segmental artery are identified along the upper edge of the interlobar artery. The posterior pulmonary artery branches and lingular segmental artery are encircled with a vessel loop and divided using a robotic stapler in order. If the pulmonary artery branch is too small and short for a stapler, the robotic Hem-o-Lok clips can be used to ligate it.
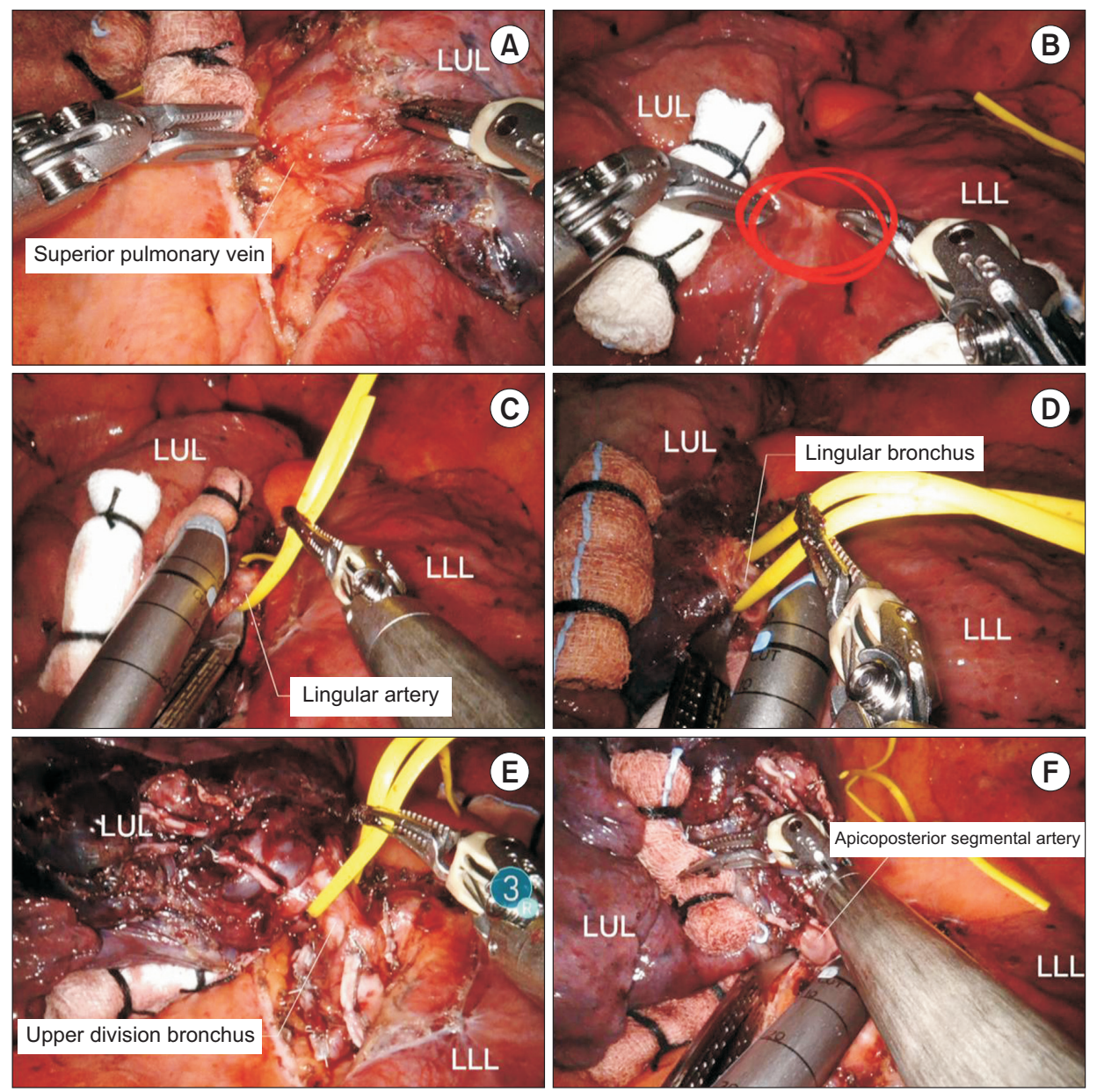

Fig. 8. Robotic left upper lobectomy. (A) Isolation of the superior pulmonary vein. (B) Dissection of the fissure. (C) Division of the lingular segmentral artery. (D) Division of the lingular bronchus. (E) Division of the upper division bronchus. (F) Division of the apcioposterior segmentral artery. LUL, left upper lobe; LLL, left lower lobe. 
Step 3: Isolation and division of the LUL bronchus

The upper lobar bronchus is located in the middle of the major fissure, and it is usually exposed after division of the lingular segmental arteries and posterior pulmonary artery branches. The lung is retracted anteriorly and the nodal tissues are removed. The normal LUL bronchus is divided into the upper division bronchus and the lingular bronchus, and they can be divided in 1 step or 2 steps. We prefer a 2-step division, in which the lingular bronchus and the upper division bronchus are divided using a robotic stapler in order.

Step 4: Isolation and division of the apicoposterior segmental artery

The apicoposterior segmental artery arises from the posterosuperior aspect of the left pulmonary artery and passes through the front of the LUL bronchus. The apicoposterior segmental artery is clearly exposed after division of the LUL bronchus. The apicoposterior segmental artery and remaining posterior pulmonary artery branches are isolated and divided using a robotic stapler in order. When the posterior pulmonary artery branches are multiple and small, they can be ligated using robotic Hem-o-Lok clips. Next, the specimen is removed using a specimen bag via the utility incision.

\section{Left lower lobectomy}

Left lower robotic lobectomy is performed in a manner similar to that of right lower lobectomy. When the fissure is well developed, we prefer a caudal approach (Fig. 9): (1) division of the inferior pulmonary ligament, (2) isolation and division of the inferior pulmonary vein, (3) isolation and division of the lower lobe bronchus, and (4) isolation and division of the inferior pulmonary artery. If the fissure is fused and not well developed, we prefer the order described in the section describing right lower lobectomy.

Step 1: Division of the inferior pulmonary ligament

The lung is retracted superiorly using Cadiere forceps and the surgeon begins to divide the inferior edge of the inferior pulmonary ligament using long bipolar forceps. An assistant can provide superior traction of the lung us-
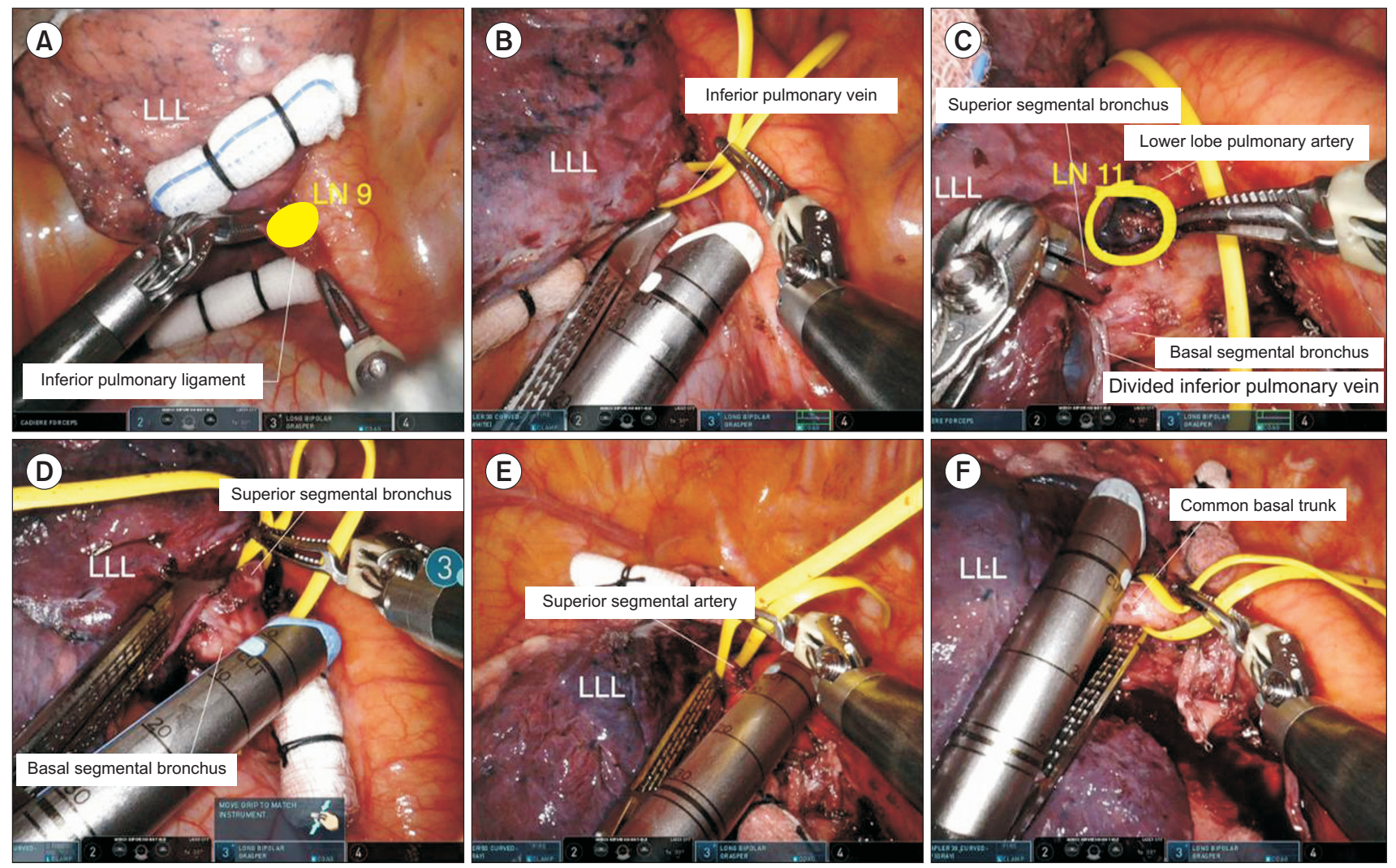

Fig. 9. Robotic left lower lobectomy. (A) Division of the inferior pulmonary ligament. (B) Division of the inferior pulmonary vein. (C) Identification of the anatomy of the pulmonary artery. (D) Division of the superior segmental bronchus. (E) Division of the superior segmental artery. (F) Division of the common basal trunk. LUL, left upper lobe; LLL, left lower lobe; LN, lymph node. 
ing a long sponge stick. The inferior pulmonary ligament is divided with all surrounding tissues, and lymph node stations 7,8 , and 9 can be removed.

Step 2: Isolation and division of the inferior pulmonary vein Once the inferior pulmonary vein is exposed, the hilum is dissected anteriorly to identify the anatomy of the pulmonary vein. The bifurcation of the inferior pulmonary vein and superior pulmonary vein should be exposed and identified. The inferior pulmonary vein is encircled with a vessel loop, and then divided using a robotic stapler.

Step 3: Isolation and division of the lower lobe bronchus

The lung is retracted anteriorly, and the left lower bronchus is dissected. The left lower bronchus divides into a superior segmental bronchus and 4 basal segmental bronchi. All hilar lymph nodes are removed, and the superior segmental bronchus is identified. Completion of the fissure can be performed using a robotic stapler, which facilitates visualization of the bronchus and artery. The left lower bronchus can be divided by 1 step or 2 steps, and we prefer a 2-step division. The superior segmental bronchus and basal segmental bronchus can be divided using a robotic stapler in order.

Step 4: Isolation and division of the lower lobe pulmonary artery

After division of the left lower bronchus, the fissure is dissected to identify the anatomy of the pulmonary artery. The left lower pulmonary artery divides into the superior segmental artery and the common basal trunk. Once the superior segment and basal trunk are exposed, they can be divided in 1 step or 2 steps. We prefer a 2-step division, wherein these structures are divided using a robotic stapler in order. Next, the remainder of the fissure is divided using a robotic stapler. Subsequently, the specimen is removed using a specimen bag via the utility incision.

\section{Conclusion}

Herein, we have presented a review of robotic lobectomy, focusing on several key technical points for a successful operation. Robotic lobectomy is feasible and safe, and it may be useful for more challenging surgical procedures. Although the learning curve for robotic lobectomy is steep, the technology of robotic surgical systems is still evolving, and upgraded systems will enhance the speed, accuracy, and safety of robotic lobectomy in the future.

\section{Conflict of interest}

No potential conflict of interest relevant to this article was reported.

\section{ORCID}

Jun Hee Lee: https://orcid.org/0000-0002-6592-6483

Jeong In Hong: https://orcid.org/0000-0002-9660-7063

Hyun Koo Kim: https://orcid.org/0000-0001-7604-4729

\section{References}

1. Yang CF, Sun Z, Speicher PJ, et al. Use and outcomes of minimally invasive lobectomy for stage I non-small cell lung cancer in the National Cancer Data Base. Ann Thorac Surg 2016;101:1037-42.

2. Emmert A, Straube C, Buentzel J, Roever C. Robotic versus thoracoscopic lung resection: a systematic review and meta-analysis. Medicine (Baltimore) 2017;96:e7633.

3. O'Sullivan KE, Kreaden US, Hebert AE, Eaton D, Redmond KC. A systematic review and meta-analysis of robotic versus open and video-assisted thoracoscopic surgery approaches for lobectomy. Interact Cardiovasc Thorac Surg 2019;28:526-34.

4. Linsky P, Wei B. Robotic lobectomy. J Vis Surg 2017;3:132.

5. Kneuertz PJ, D'Souza DM, Richardson M, Abdel-Rasoul M, Moffatt-Bruce SD, Merritt RE. Long-term oncologic outcomes after robotic lobectomy for early-stage non-small-cell lung cancer versus video-assisted thoracoscopic and open thoracotomy approach. Clin Lung Cancer 2020;21:214-24.

6. Roviaro G, Rebuffat C, Varoli F, Vergani C, Mariani C, Maciocco M. Videoendoscopic pulmonary lobectomy for cancer. Surg Laparosc Endosc 1992;2:244-7.

7. Berfield KS, Farjah F, Mulligan MS. Video-assisted thoracoscopic lobectomy for lung cancer. Ann Thorac Surg 2019;107:603-9.

8. McKenna RJ Jr. Lobectomy by video-assisted thoracic surgery with mediastinal node sampling for lung cancer. J Thorac Cardiovasc Surg 1994;107:879-82.

9. Landreneau RJ, Mack MJ, Hazelrigg SR, et al. Video-assisted thoracic surgery: basic technical concepts and intercostal approach strategies. Ann Thorac Surg 1992;54:800-7.

10. Lin J. Robotic lobectomy: revolution or evolution? J Thorac Dis 2017;9:2876-80.

11. Park BJ, Flores RM, Rusch VW. Robotic assistance for video-assisted thoracic surgical lobectomy: technique and initial results. J Thorac Cardiovasc Surg 2006;131:54-9.

12. Ricciardi S, Cardillo G, Zirafa CC, Davini F, Melfi F. Robotic lobectomies: when and why? J Vis Surg 2017;3:112.

13. Melfi FM, Menconi GF, Mariani AM, Angeletti CA. Early experience with robotic technology for thoracoscopic surgery. Eur J Car- 
diothorac Surg 2002;21:864-8.

14. Zirafa CC, Romano G, Key TH, Davini F, Melfi F. The evolution of robotic thoracic surgery. Ann Cardiothorac Surg 2019;8:210-7.

15. Galetta D, Casiraghi M, Pardolesi A, Borri A, Spaggiari L. New stapling devices in robotic surgery. J Vis Surg 2017;3:45.

16. Farivar AS, Cerfolio RJ, Vallieres E, et al. Comparing robotic lung resection with thoracotomy and video-assisted thoracoscopic surgery cases entered into the Society of Thoracic Surgeons database. Innovations (Phila) 2014;9:10-5.

17. Kent M, Wang T, Whyte R, Curran T, Flores R, Gangadharan S. Open, video-assisted thoracic surgery, and robotic lobectomy: review of a national database. Ann Thorac Surg 2014;97:236-44.

18. Veronesi G, Galetta D, Maisonneuve P, et al. Four-arm robotic lobectomy for the treatment of early-stage lung cancer. J Thorac Cardiovasc Surg 2010;140:19-25.

19. Adams RD, Bolton WD, Stephenson JE, Henry G, Robbins ET, Sommers E. Initial multicenter community robotic lobectomy experience: comparisons to a national database. Ann Thorac Surg 2014; 97:1893-900.

20. Bao F, Zhang C, Yang Y, He Z, Wang L, Hu J. Comparison of robotic and video-assisted thoracic surgery for lung cancer: a propensity-matched analysis. J Thorac Dis 2016;8:1798-803.

21. Yang HX. Long-term survival of early-stage non-small cell lung cancer patients who underwent robotic procedure: a propensity scorematched study. Chin J Cancer 2016;35:66.

22. Qiu T, Zhao Y, Xuan Y, Jiao W. Robotic-assisted double-sleeve lobectomy. J Thorac Dis 2017;9:E21-5.

23. Wilson JL, Louie BE, Cerfolio RJ, et al. The prevalence of nodal upstaging during robotic lung resection in early stage non-small cell lung cancer. Ann Thorac Surg 2014;97:1901-7.
24. Casiraghi M, Spaggiari L. Robotic lobectomy has the greatest benefit in patients with marginal pulmonary function. J Thorac Dis 2019; 11(Suppl 3):S322-4

25. Veronesi G, Novellis P, Difrancesco O, Dylewski M. Robotic assisted lobectomy for locally advanced lung cancer. J Vis Surg 2017;3:78.

26. Wei B, Cerfolio RJ. Robotic lobectomy and segmentectomy: technical details and results. Surg Clin North Am 2017;97:771-82.

27. Melfi FM, Mussi A. Robotically assisted lobectomy: learning curve and complications. Thorac Surg Clin 2008;18:289-95.

28. Gharagozloo F, Margolis M, Tempesta B, Strother E, Najam F. Robot-assisted lobectomy for early-stage lung cancer: report of 100 consecutive cases. Ann Thorac Surg 2009;88:380-4.

29. Arnold BN, Thomas DC, Bhatnagar V, et al. Defining the learning curve in robot-assisted thoracoscopic lobectomy. Surgery 2019;165: 450-4.

30. Song G, Sun X, Miao S, et al. Learning curve for robot-assisted lobectomy of lung cancer. J Thorac Dis 2019;11:2431-7.

31. Jang HJ, Lee HS, Park SY, Zo JI. Comparison of the early robot-assisted lobectomy experience to video-assisted thoracic surgery lobectomy for lung cancer: a single-institution case series matching study. Innovations (Phila) 2011;6:305-10.

32. Pearlstein DP. Robotic lobectomy utilizing the robotic stapler. Ann Thorac Surg 2016;102:e591-3.

33. Pardolesi A, Bertolaccini L, Brandolini J, Solli P, Novellis P, Veronesi G. Four arms robotic-assisted pulmonary resection-left upper lobectomy: how to do it. J Vis Surg 2018;4:109.

34. Cerfolio RJ, Bryant AS, Skylizard L, Minnich DJ. Initial consecutive experience of completely portal robotic pulmonary resection with 4 arms. J Thorac Cardiovasc Surg 2011;142:740-6. 